\title{
Author Correction: Single-cell RNA sequencing technologies and bioinformatics pipelines
}

\author{
Byungjin Hwang, Ji Hyun Lee (D) and Duhee Bang (D)
}

\section{Correction to: Experimental \& Molecular Medicine https://doi.org/10.1038/s12276-018-0071-8 published online 07 August 2018}

After online publication of this article, the authors noticed an error in the missing reference section for the Figure 3c. The correct statement of this article should have read as below.

Additional reference for Figure 3c should be added in the Figure $3 \mathrm{c}$ legend and reference section as below and original reference number through 53-103 needs to be renumbered as 54-104 since new reference ${ }^{53}$ is added: c A schematic example illustrates the difference between the
RPKM and TPM measures ${ }^{53}$. TPM is efficient for measuring relative abundance because total normalized reads are constant across different cells.

The authors apologize for any inconvenience caused.

The original article has been corrected.

Published online: 27 May 2021

\section{Reference}

53. StatQuest. "RPKM, FPKM and TPM, Clearly Explained!!!" YouTube, Joshua Starmer, 9 Jul. 2015, https://youtu.be/TTUrtCY2k-w (2015). 